\title{
IMAGE AND VIDEO TRANSMISSION OVER WIRELESS CHANNEL: A SUBBAND MODULATION APPROACH
}

\author{
H. Zheng and K. J. R. Liu \\ Department of Electrical Engineering and Institute for Systems Research \\ University of Maryland, College Park, MD 20742 \\ haitaoz,kjrliu@eng.umd.edu
}

\begin{abstract}
A new approach of reliable image and video transmission over noisy channel is proposed. The proposed subband modulation scheme combines source coding and channel modulation scheme to achieve high compression efficiency and preferable quality. Further performance gain is obtained by multiresolution modulation and bits re-mapping scheme that assign efficient mapping from source codeword to channel modulation points. The bits re-mapping scheme performs nearly same as the optimal mapping design scheme [3] but with much lower complexity. The simulation is carried out on Additive White Gaussian Noise ( $A W G N)$ channel, and extended to the slow Rayleigh fading channel.
\end{abstract}

\section{Introduction}

Reliable image and video transmission over noisy channel has been a challenge in multimedia communication, especially the transmission of large volume of data over the unreliable and bandwidth limited channels. In video conference applications the bandwidth assigned to each user is quite limited and if a scheme can reduce the bandwidth usage of each user while maintaining preferable quality, the original bandwidth can support more users and more profit can be made. Therefore, developing reliable transmission under the bandwidth constraint has become an important research issue. For subband decomposed image and video, one solution is the joint optimization of source and channel units among all the subbands.

Currently, there are two different approaches. Namely, A. combined source coding and channel coding, B. combined source coding and channel modulation. Approach A combines the source quantization and channel coding together and optimally partitions the source and channel code bits. The Rate Compatible Punctured Convolutional (RCPC) code with BPSK modulation simulated on Binary Symmetric Channel (BSC) is commonly used $[1,2]$. This is effective for very noisy channel but requires high bandwidth. Approach B does not use channel coding as protection against channel error but rather reduce the channel error effect by the use of one-to-one mapping design from source space to channel space $[3,4]$. However, since the number of constellation points is equal to the cardinality of the source codewords set, fine quantizations with large source codeword length may require large constellation set and as a result, high transmitted power.

We observe that the above two approaches fit to channels with low SNR and high SNR. When the SNR is high enough that the error probability of BPSK modulation is nearly saturated, but still not high enough for the modulations with large number of constellation points, these approaches will not achieve the best performance. In this paper we want to develop a scheme that handles the image and video transmission over noisy channel with middle range SNR (8$22 \mathrm{~dB}$ ) under tightly limited bandwidth (0.3-1.0bpp) constraint. Such condition is one of the most important performance region in practice, and yet few approaches provide good solution so far. Combining subband coding and channel modulation together, the proposed system optimally partitions the transmission bandwidth among a set of source quantizers and modulators. We develop a bit re-mapping scheme which performs nearly same as the optimal mapping design scheme in [3] but with much less computational complexity.

\section{Subband Modulation}

The combined source and channel coding approach is a classical solution to the transmission over noisy channel which has been studied extensively. It is equivalent to distributing the given bit budget efficiently among a set of given admissible source coding unit choices and channel coding unit choices, to minimize overall distortion. For subband decomposed source data, this shows more promising advantages. The source data is divided into a set of subbands with different percepture importance which can be classified by the variance/energy. Subbands with higher energy are more important since they carry more "information". The system can adjust the distortion of 
each subband by two means: (a) adjust the total rate of this subband or, (b) adjust the source to channel rate ratio of this subband. To make the adjustment easier and effective, the source coding unit and channel coding unit must be selected according to transmission requirements and channel conditions. Particularly, if transmission bandwidth is limited, both the source coding and the channel coding unit must carry efficient compression capability as well as the error protection capability. Our design is divided into three steps.

1. Select Choice of Source Unit: Different quantization will yield different bit rates and distortions. Variable length or fixed length code can be used to further code the quantized data. Variable length code has to rely on the synchronization and the distortion performance is hard to measure. Thus, we propose fixed length code which is robust and easy implemented.

2. Select Choice of Channel Unit: Roughly, the channel coding unit is a process of redundancy insertion that protects the source against channel noise. Adding channel protection codes is required for very noisy channel at cost of less compression. Indeed, for wireless communications, the channel bandwidth is the most precious resource. Under this situation, we recommend multilevel modulations which offer flexible compression capabilities as well as various error protections $[3,5]$.

3. Match Source Unit to Channel Unit: A mapping from source codeword to channel constellation point(s) has to be carefully designed. It was already shown in [3] that non-proper or random mapping will cause severe performance degradations. Multilevel modulation displays another benefit, which naturally come from the flexibility of adjusting both the constellation points distribution and the mapping from source space to channel space.

\subsection{Distortion Measure}

Using Mean Squared Error (MSE) as the distortion measure, the overall distortion can be divided into source distortion $D_{i, s}$ and channel distortion $D_{i, c}$ for each subband $i, i \in[1, M]$. Without loss of generality, the distortion that caused by both source and channel is ignored[1,2]. $D_{i, s}$ and $D_{i, c}$ can be interpreted as the function of source coding rate $R_{i, s}$ and channel modulation rate $R_{i, c}$ :

$$
D_{i, s}\left(R_{i, s}\right)=\sum_{x=0}^{N_{i, s}-1} D\left(x, x_{q}\right),
$$

$D_{i, c}\left(R_{i, s}, R_{i, c}\right)=\sum_{x_{q}=0}^{N_{i, s}-1} C_{i, x_{q}} \sum_{x_{q}^{\prime}=0}^{N_{i, s}-1} P\left(x_{q}^{\prime} \mid x_{q}, i\right) D\left(x_{q}, x_{q}^{\prime}\right)$

where $x$ and $x_{q}$ represent the source data and its quantized version, respectively. $N_{i, s}$ is the subband size and $C_{i, x_{q}}$ is number of $x_{q} . D(a, b)$ is measured as MSE between $a$ and $b$. For subband decomposed data, the overall distortion and rate are the sum of that of all the independent subbands given as

$$
D_{\text {total }}=\sum_{i=1}^{M} D_{i, s}+D_{i, c}, \quad R_{\text {total }}=\sum_{i=1}^{M} R_{i, s} / R_{i, c} .
$$

Given a bandwidth constraint $R_{b u d g e t}$ to code $M$ subbands, the objective of our approach is to find optimal rate partition $\mathcal{P}=\left\{\left(R_{i, s}, R_{i, c}\right), i=1 \ldots M\right\}$,to minimize the overall distortion, which is a constrained optimization problem defined as

$$
\min _{\mathcal{P}} D_{\text {total }} \text { subject to } R_{\text {total }}<R_{\text {budget }} \text {. }
$$

The solution is developed similarly to that of [6]. First the constrained problem can be reduced to an equivalent unconstrained problem through the Lagrange multiplier $\lambda$. For a given $\lambda$, the optimization can be solved for each subband through integer programming technique. Final solution is obtained through finding the optimal $\lambda$ that qualifies the constraint using bisection method.

\subsection{MR modulation}

The modulation design aims to find modulation schemes with efficient power consumption, high compression and reliable error protection. The multiresolution (MR) modulation proposed by [4] showed that great difference in error protection of each bit at different locations of modulation points. This observation becomes a major inspiration to our system. Figure 1 illustrates the MR modulation constellation of PAM4, QAM-16, and QAM-64. $d 2$ and $d 1$ represent intracloud and intercloud distances, respectively. We refer to each constellation point as a symbol. The MSBs of a symbol receive better protection than LSBs. It must be emphasized that the indices of codewords generated by quantizer must be arranged in a order such that if two codewords are the nearest neighbor, the original source data they represent also have the smallest mean squared difference. We provide numerical comparisons between the MR modulations and the commonly used modulations which are equivalent to the MR modulations at $\mu=1.0$. The BERs are shown in Figure 2. MR modulations will arrange the distance among the constellation points by adapting 
$\mu$ or changing the modulation rate, to reduce the total distortion. The distance between the symbols with large distortion is increased, and to maintain the same power the distance between two symbols with small distortion is decreased.

\subsection{Mapping Design}

In [4], one source codeword is directly mapped to one modulation symbol. However, for channels with middle SNR, the source codeword length is larger than the modulation rate so that one codeword may map to several constellation points, called constellation vector. Therefore, we want to develop an efficient, intelligent mapping from source codeword to constellation vector to achieve minimum channel noise effect.

\section{- The Optimal Mapping Design.}

Mathematically, the optimal mapping design can be interpreted as another optimization problem defined as follow.

Given $p\left(u \mid u^{\prime}\right), u, u^{\prime} \in \Omega, \quad x, x^{\prime} \in \mathcal{C}$, find $F$ : $\mathcal{C} \longrightarrow \Omega^{m}, \quad F(x)=\left\{u_{1}, u_{2}, . ., u_{m}\right\}$ such that

$$
\begin{gathered}
\operatorname{Min}\left\{\sum_{x, x^{\prime} \in \mathcal{C}} P\left(x^{\prime} \mid x\right) d\left(x^{\prime}, x\right)\right\} \\
=\operatorname{Min}\left\{\sum_{x, x^{\prime} \in \mathcal{C}} \prod_{i=1}^{m} p\left(u_{i}^{\prime} \mid u_{i}\right) d\left(x^{\prime}, x\right)\right\},
\end{gathered}
$$

where $\Omega$ represents the set of constellation points, $\Omega_{m}$ represents the $m$ dimensional vector extension of $\Omega$, and $\mathcal{C}$ is the set of source codewords with cardinality of $2^{R_{i, s}}$. Dynamic programming and simulated annealing can be used to find the optimal solution, but difficult and computationally expensive. Here we propose a simple, but efficient scheme.

\section{- Bit Re-mapping Scheme.}

It is based on the property that MSBs of each modulation symbol carry better error protection than LSBs in multilevel modulation. If we map the source codeword to constellation points following the original order, the order of bits inside each constellation point may be totally random unless $R_{i, s}$ and $R_{i, c}$ are integer multiple of the other one. This will cause degradation since the MSBs of the source codeword could map to the LSBs of the constellation point, thus receive less protection. To prevent this, the bits order of source codeword or the mapping has to be rearranged according to $R_{i, s}$ and $R_{i, c}$. Below, we describe the bits re-mapping scheme:

- Case 1: For $R_{i, s}<R_{i, c}$, follow the original order.
- Case 2: For $R_{i, s}>R_{i, c}$, divide the source codewords of length $R_{i, s}$ to $R_{i, c}$ group, each containing $\left\lfloor R_{i, s} / R_{i, c}\right\rfloor$ bits. Select one bit from each group and map to the same location of each group consecutively.

For example, when $R_{i, s}$ equals to 8 and $R_{i, c}$ equals to 2 , the source data is from bit 7 to bit 0 with bit 7 as the MSB and bit 0 as the LSB. There is no residue bits. Modulated following the original order, the bits order turns out to be 76 , $54,32,10$. As such bits $7,5,3$ and 1 receive more protection since they are mapped to MSB of each symbol. This is not appropriate since bit 6 is more important than bit 5,3 and 1 . After the bits re-mapping the order will be $73,62,51,40$. Bits 7, 6, 5, and 4 receive higher protection at the cost of less protection for bits $3,2,1$ and 0 . As we will see, the bit re-mapping scheme performs nearly same as optimal mapping design scheme, but with much lower complexity.

\section{AGWN Channel Simulation}

In the simulation, we use subband based encoder operating in "intraframe only" mode, which aims to prevent the error propagation. The image is 2D subband decomposed using orthogonal wavelet filters, while every two consecutive video frames are $3-\mathrm{D}$ subband decomposed together using Harr filtering in time domain and wavelet filtering in spatial domain. Targeted average bit rates range from 0.3 bit per pixel(bpp) to $1.0 \mathrm{bpp}$. The lowest subband is quantized using Lloyd Quantizer (LQ) while the other subbands are Vector Quantized (VQ). The available modulations are BPSK, MR-PAM4, MR-QAM16 and MR-QAM64 as same as those illustrated in Figure 1.

Figure 3 plots a comparison of our system without mapping design, with bits re-mapping and with optimal mapping design. Here the system without mapping design represents the direct mapping but still transmitting the residue bits separately. The optimal mapping is obtained as the optimal solution of (5). As can be seen, the bits re-mapping system performs nearly the same as the optimal mapping system and is about $0.2-2.0 \mathrm{~dB}$ better than the system without mapping design, particularly in the middle SNR range. Examining the visual quality of our system and the BPSK system from Figure 5, our resulted image is clearer and more detailed with only a negligible number of defective points. Figure 4 shows that our system outperforms one-to-one mapping system [3] for SNR range from 8-22dB. 


\section{Extension to Slow Rayleigh Fading Channel}

Although above simulation only involves AWGN channel, our system can be applied to slow Rayleigh fading channel. In [7], the adaptive modulation scheme based on switching levels is applied to slow fading channel. The transmitter and receiver are able to adjust the modulation type according to channel condition. The optimal switching levels are computed based on the received SNR value. The object function is given by

$$
\begin{gathered}
\text { Dist }=\int_{0}^{l_{1}} D_{0}(r) F(r, R) d r+\int_{l_{1}}^{l_{2}} D_{1}(r) F(r, R) d r \\
+\int_{l_{2}}^{l_{3}} D_{2}(r) F(r, R) d r+\int_{l_{3}}^{\infty} D_{3}(r) F(r, R) d r, \quad \text { (6) }
\end{gathered}
$$

where $F(r, R)$ is the probability distribution function (PDF) of the received channel SNR, $R$ is the average channel SNR and $D_{i}$ is the distortion corresponding to the quantization and modulation scheme $\left(R_{s}, R_{c}\right)$ selected for the $i$ th SNR range. For each range, $\left(R_{s}, R_{c}\right)$ can be selected as the selection used by the middle SNR value. Or the one that minimizes the total distortion summing over all SNRs within this range. For slow Rayleigh fading channel[8], the channel SNR has the PDF of $F(r, R)=\frac{1}{R} \exp \frac{-r}{R}$, where $R$ is the average channel SNR. For integer SNR (dB) value, denoted as $k$, the probability of received SNR being $k$ $\mathrm{dB}$ is $P(k)=\int_{k-0.5}^{k+0.5} F(x, R) d x$. Given the average channel SNR $R$, the optimization function is defined as

$$
\begin{aligned}
\text { Dist } & =\sum_{0}^{l_{1}-1} D_{0}(r) P(r)+\sum_{l_{1}}^{l_{2}-1} D_{1}(r) P(r) \\
+\quad & \sum_{l_{2}}^{l_{3}-1} D_{2}(r) P(r)+\sum_{l_{3}}^{\infty} D_{3}(r) P(r) .
\end{aligned}
$$

The optimal levels $l_{1}, l_{2}, l_{3}$ can be solved by dynamic programming. Table 1 and 2 shows the optimal levels that are computed for "Salesman" sequence at rate $0.5 \mathrm{bpp}$ with different average channel SNR values noted as $\mathrm{R}$, based on our system and the BPSK modulation system mentioned before.

Table 1. Simulation Result of Our Adaptive System

\begin{tabular}{|c||ccc||c|c|}
\hline $\mathrm{R}$ & level 0 & level 1 & level 2 & MSE & PSNR \\
\hline 5 & 2 & 5 & 18 & 154.28 & 26.25 \\
\hline 10 & 8 & 12 & 25 & 97.37 & 28.25 \\
\hline 12 & 8 & 12 & 25 & 77.19 & 29.26 \\
\hline 15 & 8 & 12 & 25 & 51.77 & 30.99 \\
\hline 18 & 8 & 12 & 25 & 33.67 & 32.86 \\
\hline 20 & 8 & 12 & 25 & 25.35 & 34.09 \\
\hline
\end{tabular}

Table 2. Simulation Result of BPSK Adaptive System

\begin{tabular}{|c||ccc||c|c|}
\hline $\mathrm{R}$ & level 0 & level 1 & level 2 & MSE & PSNR \\
\hline 5 & 2 & 4 & 11 & 155.30 & 26.22 \\
\hline 10 & 2 & 4 & 19 & 102.91 & 28.00 \\
\hline 12 & 2 & 6 & 13 & 92.26 & 28.48 \\
\hline 15 & 2 & 8 & 11 & 83.16 & 28.93 \\
\hline 18 & 2 & 4 & 17 & 77.92 & 29.22 \\
\hline 20 & 2 & 5 & 13 & 76.08 & 29.32 \\
\hline
\end{tabular}

As can be seen from Table 1, generally the switch level $(8 \mathrm{~dB}, 12 \mathrm{~dB}, 25 \mathrm{~dB})$ works well for all average channel SNR values. And our system performs better than BPSK modulation system especially for middle average $\mathrm{SNR}$ values $(10-20 \mathrm{~dB})$.

\section{Conclusion}

We have proposed a combined source coding and channel modulation scheme for image and video transmission over noisy channel. The flexibility of combined source and channel design as well as various compressions and UEP protections of multilevel MR modulations are fully utilized. The system performs significantly better than other schemes proposed in the literatures on the AWGN channel and the slow Rayleigh fading channel with middle range SNR and low bandwidth. Compared to conventional schemes, our scheme need 3 or 4 modulators/demodulators. Since we use common modulations such as BPSK, PAM and QAM which are quite the same in terms of implementation, the increase in complexity is ignorable compared to the performance improvement.

\section{References}

[1] G. Cheung and A. Zakhor, "Joint Source/Channel Coding of Scalable Video over Noisy Channel", Proc. ICIP,pp. 767-770,1996.

[2] M. Srinivasan, P. Burlina and R. Chellappa, "Adaptive Source-Channel Subband Video Coding for Wireless Channels", in Proc. of the First IEEE Workshop on Multimedia Signal Processing,June 1997.

[3] J. M. Lervik and T. A. Ramstad, "Robust Image Communication using Subband Coding and Multilevel Modulation", SPIE VCIP 96,pp.524535,1996 .

[4] I. Kozintsev and K. Ramchandran, "Multiresolution Joint Source-Channel Coding Using Embedded Constellations for Power-Constrained TimeVaring Channels ", Proc. ICIP, pp. 2343-2346, 1996.

[5] G. Ungerboeck, "Channel coding with multilevel/phase signals", IEEE Trans. on Information Theory, pp. 55-66, 1982.

[6] K. Ramchandran and M. Vetterli "Best Wavelet Packet Bases in a Rate-Distortion Sense", IEEE 


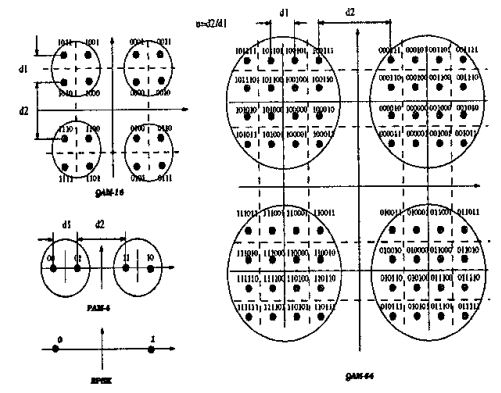

Figure 1: MR modulations

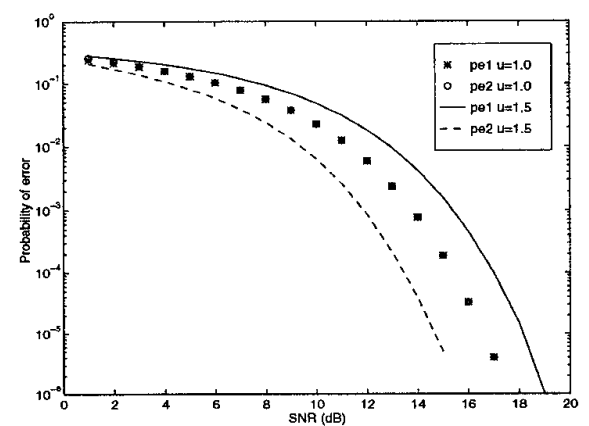

Figure 2: The BER vs SNR performance comparison between MR-PAM4 modulations and common PAM4. pe1 and pe2 stand for BER of LSB and MSB.

Trans. on Image Processing ,pp.160-174, Vol. 2, No. 2, April 1993.

[7] J.M. Tprrance and L. Hanzo, "Optimization of Switching Levels for Adaptive Modulation in a Slow Rayleigh Fading Channel", Electronics Letters, June 1996, pp 1167-1169.

[8] R. Steele, Mobile Radio Communications, IEEE Press, 1992.

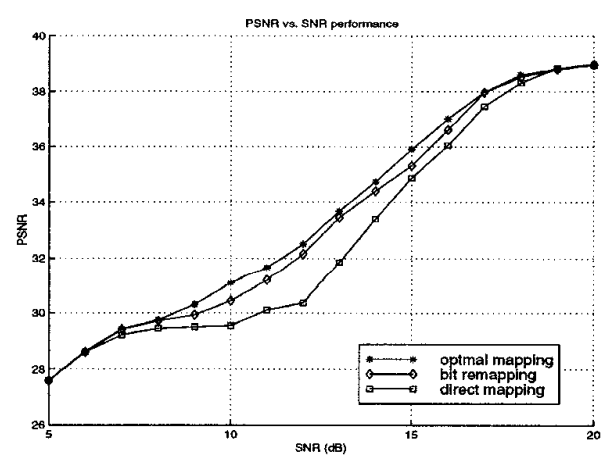

Figure 3: PSNR vs. SNR performance comparison of different mapping designs using "Salesman" sequence frame 3.

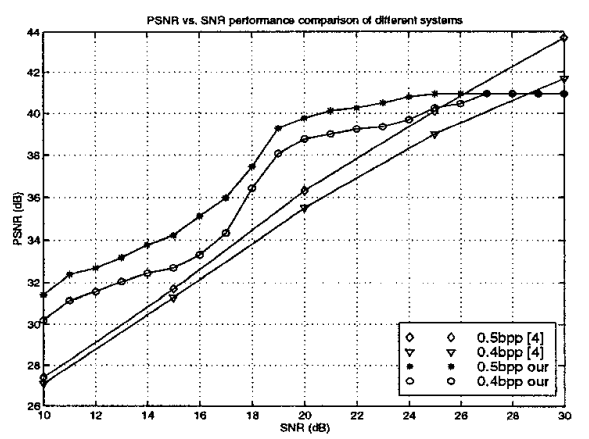

Figure 4: Comparison of our system and intelligent mapping system of [3] at different rates.

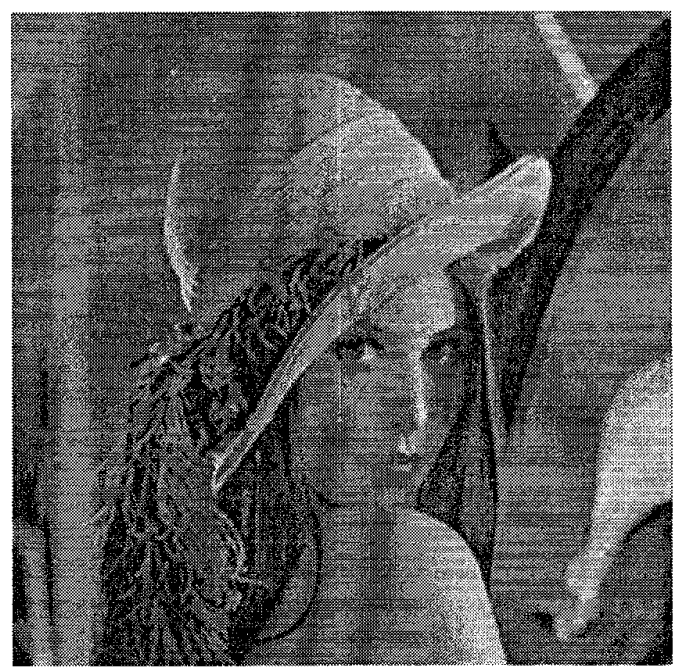

(a)

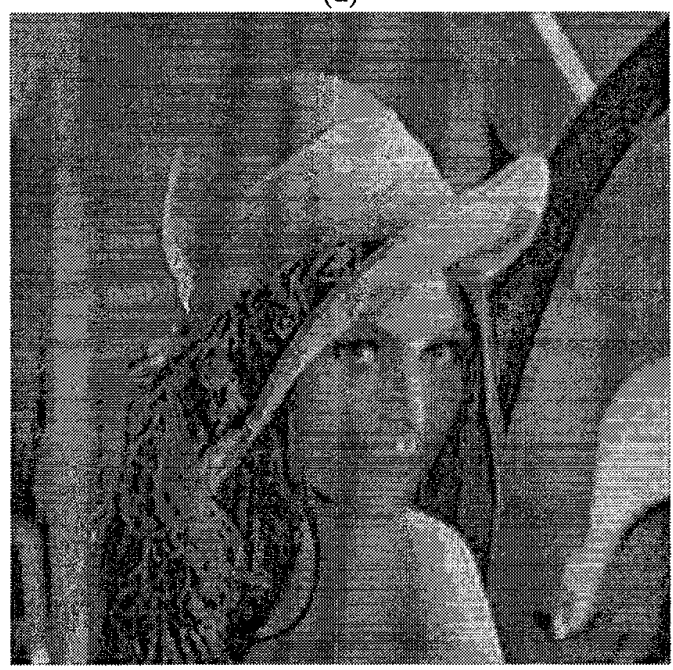

(b)

Figure 5: Image results of our system (a) and fixed BPSK modulation system (b) at 0.3bpp, $S N R=15 d B$ 